\title{
Reliability analysis of wireless communication based on ZigBee Liang-lu YIN ${ }^{1,}$ a , Yuan-liang HUANG ${ }^{1,2 b}$, and Wei-xiong CHEN ${ }^{1}$ \\ ${ }^{1}$ Institute of electrical automation, Jinan University, Guangdong, Zhuhai519070, china; \\ ${ }^{2}$ Institute of rail transport, Jinan University, Guangdong, Zhuhai519070, china \\ a15820578840@163.com, btyoll@jnu.edu.cn`
}

Keywords: ZigBee; Reliability ; RSSI; PER; Pseudo loss.

\begin{abstract}
On the basis of analyzing the development status of the ZigBee technology, a new method to analyze the wireless communication reliability is proposed by using the signal strength (RSSI), through the formation of a wireless network model by using module CC2530PA, all the packets received in the ZigBee wireless network technology are collected, and then the relational model of RSSI and PER is constructed, the "pseudo-loss" phenomenon is analyzed, and the RSSI threshold based on the wireless network reliability is determined, which is $-75 \mathrm{dBm}$, At last, on the reliability of wireless communication during data transmission, the results have very high practical value and promoting function in the ZigBee technology in the future.
\end{abstract}

\section{Introduction}

ZigBee, an emerging technologyin the wireless communication field, raises great concerns of the academic and commercial areas. The ZigBee reliability analysis is always the focus. Many domestic and foreign researchers are doing research on ZigBee reliability from a single factor.[1][7] However, this article reflects the impact of a number of factors by signal strength, using the CC2530PA module to form a simple ZigBee networking model, collecting all the packets the coordinator received at the computer, and through theanalysis of data, relationships between the RSSI with symbol error rate and packet loss rate are determined.Considering theRSSI reliability threshold and the specific needs of data transmission reliability, the ideal distance ofnode in positioning applications can be drawn.[1][2][6]

\section{Platform Design}

The hardware used is TI's module CC2530PA, the core is CC2530, The software design are under TI's integrated development environment, provided by IAR.[3][4]

The experiments are carried out in the open field, detecting communication resultsby changing the distance between the coordinator and the terminal device.This study uses a simplecommunication model, it has a plurality of nodes, a coordinator, which receives information. The other nodes are terminal devices.[7]

The terminal device sends the symbol error rate packet when detecting the symbol error rate. In experiments, when the packets received at a certain distance are closing to 100, the next round of collection begin. In the collectingprocess, the two adjacent interval distance slowly decreases. When detecting the packet loss rate, the terminal device sends the packet loss rate packet, this time, at each distance, we must ensure that received packetsare nearly 1000.[5]

\section{Signal Analysis}

Signal Strength Characterization:Received signal strength indication, RSSI, is a measure for the signal strength, the larger the RSSI is,stronger the signal is.[7]

Symbol Error Rate: Symbol error rate, SER, refers to the percentage of the number of error transmitted symbols within the total symbol transmission time, BER is the index to measure the accuracy of the data transmission.[1] 
Packers Error Rate: Packers error rate, PER, refers to the percentage of the number of packets that the recipient does not receive within the total number of packets sent by the sender. PER reflectsthe transmission reliability.[1]

Pseudo-loss: In "Pseudo-loss" phenomenon, data packets are not truly lost, but the disorder phenomenon occurs, the pseudo-loss rate is a special factor of reflection.

\section{The analysis of wireless network transmission reliability}

BER Analysis: In order to improve the accuracy, in the same distance, the data packets are collected in chronological order. 10 packets is a group, a group is a data packet, and the last set of data is invalid.Then,numbers of symbols in each data packet to be detected reach 960, the corresponding signal strength is the average of 10 packets. Then the group obtained 283 valid data, as shown in Table 1, in these pairs, eithercorresponding BERvalue are $0.00 \%$, so there is no symbol errors phenomenon.

Table1: The data of RSSI and SER

\begin{tabular}{|c|c|c|c|c|c|c|c|c|c|c|c|c|c|}
\hline RSSI & $\begin{array}{c}\text { SER } \\
{[\%]}\end{array}$ & RSSI & $\begin{array}{l}\text { SER } \\
{[\%]}\end{array}$ & RSSI & $\begin{array}{l}\text { SER } \\
{[\%]}\end{array}$ & RSSI & $\begin{array}{l}\text { SER } \\
{[\%]}\end{array}$ & RSSI & $\begin{array}{l}\text { SER } \\
{[\%]}\end{array}$ & RSSI & $\begin{array}{l}\text { SER } \\
{[\%]}\end{array}$ & RSSI & $\begin{array}{l}\text { SER } \\
{[\%]}\end{array}$ \\
\hline-54.1 & 0.00 & -65.8 & 0.00 & $\begin{array}{l}-73.7 \\
\end{array}$ & 0.00 & -75 & 0.00 & $\begin{array}{l}-76.5 \\
\end{array}$ & 0.00 & $\begin{array}{l}-78.8 \\
\end{array}$ & 0.00 & -80.7 & 0.00 \\
\hline-55.2 & 0.00 & -65.9 & 0.00 & -73.8 & 0.00 & -75 & 0.00 & -76.9 & 0.00 & -78.8 & 0.00 & -80.8 & 0.00 \\
\hline-55.4 & 0.00 & -65.9 & 0.00 & -73.8 & 0.00 & -75 & 0.00 & -76.9 & 0.00 & -78.8 & 0.00 & -80.8 & 0.00 \\
\hline-55.7 & 0.00 & -66 & 0.00 & -73.9 & 0.00 & -75 & 0.00 & -77 & 0.00 & -78.9 & 0.00 & -80.8 & 0.00 \\
\hline-55.7 & 0.00 & -66.1 & 0.00 & -74 & 0.00 & -75 & 0.00 & -77 & 0.00 & -78.9 & 0.00 & -80.9 & 0.00 \\
\hline-56.1 & 0.00 & -66.1 & 0.00 & -74 & 0.00 & -75.1 & 0.00 & -77 & 0.00 & -78.9 & 0.00 & -80.9 & 0.00 \\
\hline-56.5 & 0.00 & -66.5 & 0.00 & -74 & 0.00 & -75.1 & 0.00 & -77.1 & 0.00 & -79 & 0.00 & -81 & 0.00 \\
\hline-56.6 & 0.00 & -66.6 & 0.00 & -74.5 & 0.00 & -75.1 & 0.00 & -77.1 & 0.00 & -79.1 & 0.00 & -81.1 & 0.00 \\
\hline-56.6 & 0.00 & -66.7 & 0.00 & -74.5 & 0.00 & -75.1 & 0.00 & -77.1 & 0.00 & -79.1 & 0.00 & -81.1 & 0.00 \\
\hline-58.2 & 0.00 & -66.9 & 0.00 & -74.6 & 0.00 & -75.1 & 0.00 & -77.2 & 0.00 & -79.1 & 0.00 & -81.1 & 0.00 \\
\hline-58.6 & 0.00 & -67 & 0.00 & -74.7 & 0.00 & -75.1 & 0.00 & -77.2 & 0.00 & -79.2 & 0.00 & -81.1 & 0.00 \\
\hline-58.7 & 0.00 & -67.1 & 0.00 & -74.7 & 0.00 & -75.2 & 0.00 & -77.3 & 0.00 & -79.3 & 0.00 & -81.2 & 0.00 \\
\hline-60.3 & 0.00 & -68 & 0.00 & -74.7 & 0.00 & -75.2 & 0.00 & -77.3 & 0.00 & -79.5 & 0.00 & -81.3 & 0.00 \\
\hline-61.1 & 0.00 & -68.7 & 0.00 & -74.7 & 0.00 & -75.2 & 0.00 & -77.4 & 0.00 & -79.5 & 0.00 & -81.4 & 0.00 \\
\hline-61.3 & 0.00 & -68.7 & 0.00 & -74.7 & 0.00 & -75.2 & 0.00 & -77.5 & 0.00 & -79.5 & 0.00 & -81.5 & 0.00 \\
\hline-61.7 & 0.00 & -69.7 & 0.00 & -74.7 & 0.00 & -75.2 & 0.00 & -77.7 & 0.00 & -79.5 & 0.00 & -81.6 & 0.00 \\
\hline-61.7 & 0.00 & -70.5 & 0.00 & -74.8 & 0.00 & -75.3 & 0.00 & -77.7 & 0.00 & -79.7 & 0.00 & -82 & 0.00 \\
\hline-61.8 & 0.00 & -70.6 & 0.00 & -74.8 & 0.00 & -75.3 & 0.00 & -77.8 & 0.00 & -79.7 & 0.00 & -82 & 0.00 \\
\hline-62.7 & 0.00 & -71.4 & 0.00 & -74.8 & 0.00 & -75.3 & 0.00 & -77.8 & 0.00 & -79.8 & 0.00 & -82.2 & 0.00 \\
\hline-62.7 & 0.00 & -72.4 & 0.00 & -74.8 & 0.00 & -75.3 & 0.00 & -77.9 & 0.00 & -79.8 & 0.00 & -82.2 & 0.00 \\
\hline-62.8 & 0.00 & -72.6 & 0.00 & -74.8 & 0.00 & -75.3 & 0.00 & -78 & 0.00 & -79.8 & 0.00 & -82.3 & 0.00 \\
\hline-63.3 & 0.00 & -72.6 & 0.00 & -74.8 & 0.00 & -75.4 & 0.00 & -78.1 & 0.00 & -79.8 & 0.00 & -82.3 & 0.00 \\
\hline-63.4 & 0.00 & -72.7 & 0.00 & -74.8 & 0.00 & -75.4 & 0.00 & -78.2 & 0.00 & -79.9 & 0.00 & -82.5 & 0.00 \\
\hline-63.4 & 0.00 & -72.9 & 0.00 & -74.8 & 0.00 & -75.4 & 0.00 & -78.2 & 0.00 & -80 & 0.00 & -82.6 & 0.00 \\
\hline-63.4 & 0.00 & -72.9 & 0.00 & -74.8 & 0.00 & -75.4 & 0.00 & -78.2 & 0.00 & -80 & 0.00 & -82.7 & 0.00 \\
\hline-63.7 & 0.00 & -73 & 0.00 & -74.8 & 0.00 & -75.5 & 0.00 & -78.2 & 0.00 & -80 & 0.00 & -82.8 & 0.00 \\
\hline-63.7 & 0.00 & -73 & 0.00 & -74.8 & 0.00 & -75.6 & 0.00 & -78.3 & 0.00 & -80 & 0.00 & -83.1 & 0.00 \\
\hline-64 & 0.00 & -73.1 & 0.00 & -74.8 & 0.00 & -75.6 & 0.00 & -78.3 & 0.00 & -80.1 & 0.00 & -83.1 & 0.00 \\
\hline-64.2 & 0.00 & -73.1 & 0.00 & -74.8 & 0.00 & -75.6 & 0.00 & -78.3 & 0.00 & -80.1 & 0.00 & -83.2 & 0.00 \\
\hline-64.2 & 0.00 & -73.1 & 0.00 & -74.9 & 0.00 & -75.7 & 0.00 & -78.4 & 0.00 & -80.2 & 0.00 & -83.3 & 0.00 \\
\hline-64.4 & 0.00 & -73.1 & 0.00 & -74.9 & 0.00 & -75.7 & 0.00 & -78.5 & 0.00 & -80.2 & 0.00 & -83.3 & 0.00 \\
\hline-64.4 & 0.00 & -73.2 & 0.00 & -74.9 & 0.00 & -75.8 & 0.00 & -78.5 & 0.00 & -80.2 & 0.00 & -83.6 & 0.00 \\
\hline-64.5 & 0.00 & -73.3 & 0.00 & -74.9 & 0.00 & -75.8 & 0.00 & -78.6 & 0.00 & -80.3 & 0.00 & -83.6 & 0.00 \\
\hline-64.6 & 0.00 & -73.3 & 0.00 & -74.9 & 0.00 & -75.8 & 0.00 & -78.6 & 0.00 & -80.3 & 0.00 & -83.7 & 0.00 \\
\hline-64.6 & 0.00 & -73.3 & 0.00 & -74.9 & 0.00 & -75.9 & 0.00 & -78.6 & 0.00 & -80.4 & 0.00 & -83.8 & 0.00 \\
\hline-65 & 0.00 & -73.4 & 0.00 & -74.9 & 0.00 & -76.1 & 0.00 & -78.7 & 0.00 & -80.4 & 0.00 & -84.6 & 0.00 \\
\hline-65.2 & 0.00 & -73.5 & 0.00 & -74.9 & 0.00 & -76.1 & 0.00 & -78.7 & 0.00 & -80.5 & 0.00 & -88 & 0.00 \\
\hline-65.3 & 0.00 & -73.6 & 0.00 & -74.9 & 0.00 & -76.1 & 0.00 & -78.7 & 0.00 & -80.5 & 0.00 & & \\
\hline-65.3 & 0.00 & -73.6 & 0.00 & -74.9 & 0.00 & -76.2 & 0.00 & -78.7 & 0.00 & -80.6 & 0.00 & & \\
\hline-65.3 & 0.00 & -73.7 & 0.00 & -74.9 & 0.00 & -76.3 & 0.00 & -78.7 & 0.00 & -80.6 & 0.00 & & \\
\hline-65.6 & 0.00 & -73.7 & 0.00 & -75 & 0.00 & -76.4 & 0.00 & -78.8 & 0.00 & -80.7 & 0.00 & & \\
\hline
\end{tabular}

When the RSSI value approaches -80 , the packet loss occurs, in existing data, the maximumneighboringdifference is 85 , that's when 84 packets are lost. 
In this experiment, the coordinator collected a total of 2880 packets, corresponding RSSI values range from -49 to -89 , when the RSSI is closing to -89 , it is difficult for coordination to receive data packets. In this present experiment, -89 is the worst value.

Packet loss rate analysis:When detecting packet loss rate in 21 distances, 22568 packets are collected. RSSI values range from -4 to -91 , when analyzing the packet loss rate, the 1000 data packets are regarded as a set of data.

Tthe RSSI value is an average of all the collected data. The packets number will be stored in a document, the sum numbers of the received packets $i_{1} n_{1}$.After reading a packet, the program compares the current data packet number num and the last one num_pre,through the result, we can determine whether the packet loss happens or not. Details as follows:[2]

If num=num_pre +1 or num=0 and num_pre=255, no packet loss.

If num pre $=255$ and num $\neq 0$, the number of lost packets is num.

If num_pre $<$ num $\leq 255$, the number of lost packets isnum - num_pre -1 .

If num_pre $<255$ and num=0, the number of lost packets is 256-num_pre.

If num_pre $<255$ andnum $<$ num_pre,the number of lost packets is $255-$ num_pre + num.

If the phenomenon of losing 256 or more packets does not occur, the sum number of lost packets is $\mathrm{n}_{2}$. PER is calculated by equation (1):

$\mathrm{PER}=n_{2} \div\left(n_{1}+n_{2}\right) \times 100 \%$ (1)

Pseudo packet loss phenomenon: In this experiment, the coordinator doesn't make response to the terminal reception device. Therefore, the following situations mayprobably cause the loss:

Poor wireless environment cause packet loss; the packets received exist the phenomenonof symbol error, the packet is abandoned.

The packet number.

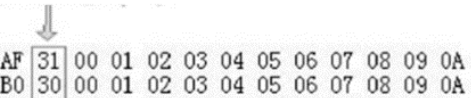

$\begin{array}{lllllllllllll}\mathrm{B} 0 & 30 & 00 & 01 & 02 & 03 & 04 & 05 & 06 & 07 & 08 & 09 & 0 \mathrm{~A}\end{array}$

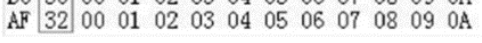

Fig.1 'Pseudo loss' phenomenon

The "pseudo-loss" phenomenon may occurs, as shown in FIG. 1.The packet No. 31, relatively lager,is received early by coordinator, but the No. 30, relatively smaller, is later. The authorinnovatively defines the "pseudo-loss" phenomenon. Which severely influenced the accuracy.

Modeling of Reliability Analysis: Some RSSI valuesare corresponded to two packet loss data. And points in the chart represents the "pseudo-loss" phenomenon, the smooth curve is the trend curve based on the line graph, as shown in FIG. 2, the fittingcurve equation:

$\mathrm{y}=1.279 \times 10^{-11} \times e^{-0.2641 x}$

The argument is RSSIvalue, and the dependent variable is PER.

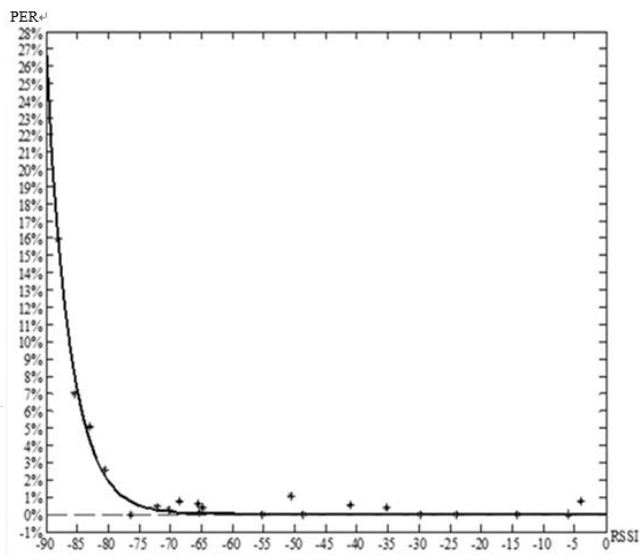

Fig.2 RSSI and packet error rate in relation line chart

In Figure 2, the packet loss occurswhen the RSSI value is greater than -75, but the overall loss rate is less than $1 \%$, when RSSI is -80 , the packet loss rate suddenly increases, and when the RSSI value gets smaller, the packet loss becomes serious, soon it shows a trendto be an exponential growth.And 
when the RSSI is about -88, the packet loss rate increases to $16 \%$.

The following are conclusions based on the "pseudo-loss" phenomenon:

When a packet is disturbed in transmission, it is possible thatlatter packets arrive earlier, whichconfuses the arriving order, then the "pseudo-loss" phenomenonhappens.

The smaller the RSSI value is, the more likely a "pseudo-loss" Phenomenon happens.

When the distance gets longer, the possibility of "pseudo-loss" should be larger, butthis time the packet loss is severe, the possibility becomes large that a packet cannot reach the recipient timely, so the "pseudo dropout" phenomenon may not appear.

\section{Conclusions}

In this paper, It can be considered that there exists no symbol error packet, and PER gets weaken when RSSI increases, when the RSSI is greater than -75 , the packet loss rate remained below $1 \%$, but when it is greater than -80 , the packet loss rate will be exponential growth. The following are recommendations for ZigBee applications:[7]

Ensure that theRSSI value between critical nodes are as large as possible, and the nodes should not be too close;

Make sure the RSSI value between nodes, which have direct communication links, is not less than -75, in specific application, the distance threshold between nodes can refer to the signal strength threshold results. In the tree topology, the children nodes do not communicate with each other, whichsubject to different parent nodes. Therefore, these child nodes are not limited by the RSSI value;

The receiver should make a feedback to the sender.When the packet number does not conform, errors should be warned, as same as other identification, and thenthe sender will be asked to resend the packet, this will avoid the packet loss, andmake a correction for the disorderof packets.

\section{References:}

[1]. Lingling. Cao: Reliability Analysis in Wireless Networks. Nanjing: Nanjing University of Aeronautics and Astronautics, 2012.

[2]. Zhihong. Qian, Dayang. Sun and L. Victor: Survey on Localization Model in Wireless Networks. Chinese Journal of Computers, 2016, 36(6) P1239-1253.

[3]. Hengjun. Zhu, Baoji. He, Hong Liang, Nannan. Zhang: Research on Optimization Method of Personnel Positioning in Mine Based on Zigbee. Computer Simulation, 2015, 32(11) P311-314.

[4]. Wenjing. Liu, Tongfei. Zhang, Zhizhen. Hu, Peng. Hu, Peng. Yang, Jingbo. Guo: A Transmission Line Monitoring Device Based on Ultrasonic and Wireless Networking Technologies. Automation of Electric Power Systems, 2013, 37(10) P98-103.

[5]. Shangbin. Jiao, Dan. Song, Qing. Zhang, Jinwei. Tang: Coal mine monitoring system based on Zig Bee wireless sensor networks. Journal of Electronic Measurement and Instrumentation, 2013, 27(5) P436-442.

[6]. Zhou. Lv: Research and Implementation of ZigBee Wireless Location Network. Wuhan: Huazhong University of Science and Technology, 2009.

[7]. Qiaoyun. Chen: Research of Link Reliability on ZigBee Wireless Sensor Network. Dalian, 2008 